\title{
Spiral Antennas for Communication Engineering Applications: A Systematic Approach
}

\author{
Shashikant Patil \\ SVKMs NMIMS Mumbai \\ sspatil@ieee.org \\ Chinmaya Vyas \\ SVKMs NMIMS Mumbai \\ chinmay.vyas109@gmail.com \\ Amar Khalore \\ SVKMs NMIMS Mumbai \\ amar.khalore@nmims.edu
}

\begin{abstract}
The field of antenna's has progressed rapidly in the recent years it can certainly be considered as the main driving force behind the advancements being accomplished in the field of communications technology specifically wireless. This paper deals with the spiral antennas and their applications in various fields their properties which on further optimisation have lead to several inventions. The spiral antenna has several advantages over the other antenna's namely easy impedance matching, superior radiation efficiency, high spectral efficiency, consistent gain, high bandwidth aids in wireless communication and their major merit being they are frequency independent hence they find their application in various fields. The design optimisation is another main issue which largely determines the performance of an antenna similarly in spiral antenna also the variation in no of turns changing the width of its arm is helpful in producing best results. The following paper gives you the insight of developments done in the field of spiral antenna and the important conclusions which can be drawn from them for further use.
\end{abstract}

Keywords: Spiral antenna, Polarisation, Archimedean spiral, Axial Ratio, Ultra wideband ratio, Feed circuitry, Unbalanced-excitation.

\section{INTRODUCTION}

Antennas have brought a revolution in the field of wireless communication systems. Wireless communication which was once a distant dream for many has now been realised with the aid of antennas. From initially being designed for the radiation and acceptance of the electromagnetic waves it has now entered phase where it optimises or accentuates the energy in some direction whereas it suppresses it in other [1].

The field of antenna has undergone a sea change specially the design, initially a system which had 50 vertical wires has now been transformed into a size of few $\mathrm{mm}$. Since the ancient antenna systems not focussed much on the designing of the antenna in the overall system analysis but now a days it is one of the most important factors while considering any antenna system, as the success and failure of the system relies heavily on the design of the antenna[2].With the design optimisation the antennas slowly and steadily have found their applications in many fields other then communication namely wearable health devices wherein the ocular sensors used as tear sensors for monitoring the glucose levels in for non invasive monitoring [3].

With the increasing importance of design optimisation we would like throw light on one of the major design development in antenna which took place early in 1956 and led to the formation of spiral antenna [4].A spiral antenna is a radio frequency antenna which holds a property of being frequency independent and thus are wideband antennas and finds its application mainly in the defence industry for sensing applications [5].Various characteristics known of a spiral antenna are as follows polarisation, radiation pattern and impedance are some other parameters which remain unchanged[8], they have a low gain and as their shape suggests they are circularly polarised. Among the class of antennas the spiral antennas may be termed as reduced sized antennas due to its windings.Design of spiral antenna can be optimised by varying number of turns it and the spacing between its turns and the width of its arm [7]. 
The gain of the spiral antenna which is initially low and the array of the spiral antennas are generated in order to increase the gain value of the spiral antenna. Spiral antennas are classified into different types; Archimedean spiral, square spiral and star spiral etc. Archimedean is the most popular amongst them because of their ability to maintain consistent gain and input impedance and excellent performance in circular polarisation [6].

These antennas operate in $3 \mathrm{~W}$ way; travelling wave, fast wave and leaky wave. The travelling wave, formed on spiral arms, allows for broadband performance, fast wave due to mutual coupling phenomenon occurring between arms of spiral and leaky wave leaks the energy during propagation through the spiral arms to produce radiation [9].

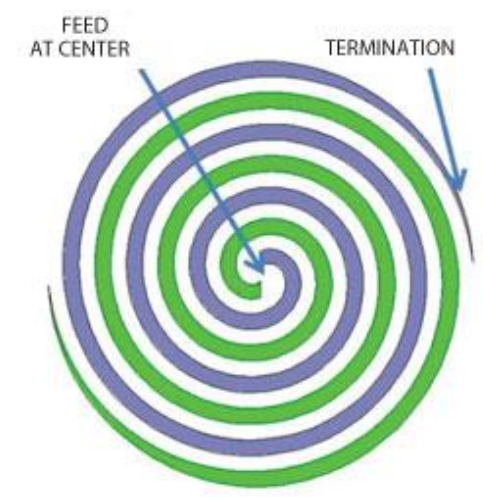

Figure1. Archimedean spiral antenna [10].
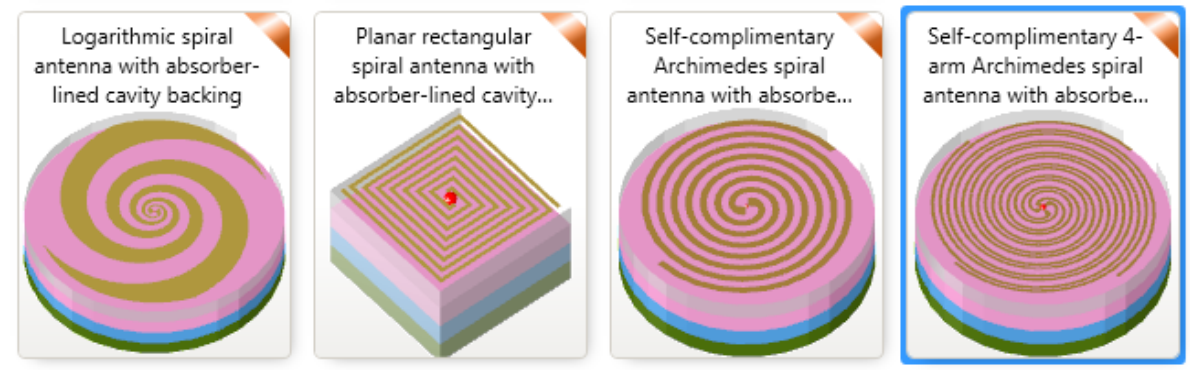

Figure2. Various types of spiral antennas [20].

\section{RESULTS AND DISCUSSIONS}

Antennas are mainly used for communication purposes, with the expansion of the wireless communication and its applications which requires larger bandwidth the need for wideband antennas also increased and thus automatically accentuating demand for spiral antennas.

We would be discussing various developments in the field of spiral antennas from their feeding methods, their application in wifi and Chinese navigation system and many others.

As per the discussion the popularity of the spiral antennas in the field of communication in pretty evident from the recent work.

Hangying Yuan et al [11] in their work showed thatDual-band antennas are more preferable in Chinese Compass Navigation Satellite System (CNSS for short), as they can provide both navigation and communication services better than any other antennas. Here, author presents dual-band dualcircular-polarized planar spiral-slot CNSS antenna. This antenna works at L band which operates on frequency of $1616 \pm 5 \mathrm{MHz}$ and is left-handed circularly polarized and at $\mathrm{S}$ band which operates on frequency of $2492 \pm 5 \mathrm{MHz}$ and is right-handed circularly polarized. In practice, results showed that the impedance bandwidth $(\mathrm{S} 11<-10 \mathrm{~dB}), 3 \mathrm{~dB}$ axial ratio bandwidth and antenna gain at $\mathrm{L}$ band are about $242 \mathrm{MHz}, 79 \mathrm{MHz}$ and $4.92 \mathrm{~dB}$, respectively, while the simulated impedance bandwidth (S11 < $-10 \mathrm{~dB}), 3 \mathrm{~dB}$ axial ratio bandwidth and antenna gain at $\mathrm{S}$ band are about $180 \mathrm{MHz}, 58 \mathrm{MHz}$ and $5.25 \mathrm{~dB}$, respectively. When an experiment was carried out for verification, measured results showed that impedance bandwidth $(\mathrm{S} 11<-10 \mathrm{~dB})$ and $3 \mathrm{~dB}$ axial ratio bandwidth $\mathrm{L}$ band are about $300 \mathrm{MHz}$ and $14 \mathrm{MHz}$, respectively, while the measured impedance bandwidth $(\mathrm{S} 11<-10 \mathrm{~dB})$ and $3 \mathrm{~dB}$ axial ratio bandwidth at $\mathrm{S}$ band are about $210 \mathrm{MHz}, 10 \mathrm{MHz}$, respectively. The measured results are almost same as the simulated ones and meet the requirement of CNSS terminal antennas. 
In this paper, it is proved that a dual-band dual-polarized spiral-slot Microstrip antenna is capable of simultaneously sending left-handed circularly polarized L-signals and receiving right-handed circularly polarized S-signals of CNSS. Fabrication of this antenna must be very precise as circular polarization characteristic is very sensitive. Antenna can be re-scaled and re-optimized to provide a compact structure and achieve high performance for different frequency sets.

Next the work of N. H. Abdul Hadiet.al [12] highlights the design, simulation, analysis and fabrication of a coaxial- fed rectangular spiral Microstrip antenna (RSMA) for Wi-Fi application. The centre frequency is $2.45 \mathrm{GHz}$ and the bandwidth is $22 \mathrm{MHz}$ The radiation pattern of this RSMA illustrated an Omni-directional pattern with voltage standing wave ratio of less than two, return loss of less than $-10 \mathrm{~dB}$, the line impedance of $50 \Omega$ and gain of $5 \mathrm{dBi}$. All the design and simulation of this RSMA were carried by using a commercial 3-D electromagnetic simulator. The prototype was fabricated on FR 4 substrate with 4.9 dielectric constant and $1.54 \mathrm{~mm}$ of thickness. Vector network analyzer ZVA40 was used to measure all the parameters of this RSMA. The 2D radiation pattern was obtained by using antenna training system ED3200. It was observed that the simulated and measured values of the parameters of the RSMA were quite close with each other.

This paper has presented the design, simulation and the prototype of RSMA, fabricated on FR4 substrates with 4.9 dielectric constant and $1.54 \mathrm{~mm}$ thickness. The results show that the simulated and measured values were quite close with each other. The relationship between $f_{c}$ and $W$ is inversely proportional. The improvement has been achieved as the size is reduced to $6 \mathrm{~cm} \times 6 \mathrm{~cm}$. The bottom coaxial fed provide space for other device to mount on the substrate which can further reduced the overall size of a Wifi.

Now the wideband applications of spiral antenna has always been advantageous to it the work proposed by Magnus Karlssonet.al [15] states that Spiral antenna possesses high spectral efficiency as compared to other antennas such as planar antennas. Thus the spiral antenna is used to support high frequencies. Since a large amount of bandwidth is required for wireless applications, wideband antennas are of at most importance. The ultra wideband radio (UWB) utilizes the frequency range of 3.1 to $10.6 \mathrm{GHz}$. So to improve performance beyond that of a single antenna, a large amount of antennas can be combined to form a new array of antenna. To extend bandwidth, two spiral antennas can be electrically coupled in a parallel way. Generally, the spiral antennas to be combined can have different radii of the radiation zone. This can result in an improved antenna performance for UWB since the standing wave ratio can be kept low for a very large bandwidth. The spiral antenna dimensions highly affect the gain and SWR.

The substrate thickness and the dissipation factor affect the antenna gain adversely. The air gap between the ground plane and antenna plane can be used for gain improvement. The dimensions therefore are the deciding factor for the antenna gain and SWR.

Now after the discussion of the applicative part there is need to discuss the feeding mechanism.

An antenna feed refers to the components of an antenna which feed the radio waves to the rest of the antenna structure, or in receiving antennas collect the incoming radio waves, convert them to electric currents and transmit them to the receiver, whereas a feed line which connects the feed antenna with the transmitter and receiver.

The planar spiral antennas have many advantages like great performance on circular polarization, easy impedance matching, and superior radiation efficiency [13].The major disadvantage of the technique being that its bandwidth is limited in circular polarisation. The vertical feeding structure has several demerits few being long length, inconsistent with the requirements of the low-profile spiral antenna geometry poor radiation behaviour, limited bandwidth [14].

Hence then came the need for parallel plane feeding structure. Hence huifen huang et.al [14] proposed the below discussed system which actually served as a remedy to the above mentioned problems.

Normally, the low-profile spiral antennas have lack of proper planer feeding ways in practical applications. During simulation, the vertical balun has a significantly long length, and doesn't meet the requirements of the low profile spiral antenna geometry. To solve this problem, a spiral antenna with integrated parallel- plane feeding structure is proposed in this paper. The antenna here used has improved axial ratio compared to the equiangular spiral antenna at low frequencies. It also moves the traditional third balun in the third dimension to the planer plane. The antenna and the Dyson-style 
balun have been integrated into a multi-layer structure and this structure maintains the typical radiation behaviour and the broadband operation of the spiral antennas. The vertical balun is also integrated at the same plane with the antenna. This largely reduces the size of antenna.

Thus the following conclusion was drawn from the research: As low-profile spiral antennas don't meet the requirements of antenna geometry, a new spiral antenna with a parallel-plane feeding structure is proposed. The antenna used has a improved axial ratio over equiangular spiral antenna. And the traditional vertical balun has been replaced by integrated parallel-plane feeding structure. The resulting structure maintains the typical radiation behaviour and broadband operation of spiral antennas. It achieves good axial ratio performance without the vertical balun. The overall size of the spiral antenna is largely reduced.

The work of H.nakano et al [15] describes a two-arm unbalanced-excitation spiral antenna, where one arm is fed by the inner conductor of a coaxial line and the other is short-circuited to a conducting cavity that backs the spiral. Analysis is performed over a frequency range of $1 \mathrm{GHz}$ to $10 \mathrm{GHz}$. The input resistance for the unbalanced-excitation spiral is smaller than that for the corresponding balanced-excitation spiral. The radiation is circularly polarized within the analysis frequency range. The unbalanced-excitation spiral exhibits a frequency response for the gain that is similar to that of the balanced-excitation spiral.

A two-arm UNB-SP is proposed as a wideband CP antenna. One of the two arms is fed by a coaxial line, with the other arm acting as a parasitic element. It is emphasized that the UNB-SP does not have a balun circuit, which is required for a conventional two-arm B-SP fed by an unbalanced feed.

The investigation reveals that the input resistance for the UNB-SP is smaller than that for the B-SP over a wide frequency range. The radiation for the UNB-SP around the $\mathrm{z}$-axis is circularly polarized. The beam direction slightly deviates from the antenna axis (z-axis). The axial ratio in the $\mathrm{z}$-direction is low, as desired. The frequency response of the gain for the UNB-SP is similar to that for the B-SP.

The antenna can be transformed to give optimum performance if certain parameters are improved. Hence the work of Huifen Huang et.al[6] describes the axial ratio characteristics of an Archimedean spiral antenna placed on a thin magnetic material an antenna which is used here has improved axial ratio (AR) and shorted arm length. The antenna which is developed is the combination of low frequency characteristic of power spiral antenna and high frequency characteristic of Archimedean spiral antenna. Archimedean spiral antenna which is used in this paper is a good choice in ultrawideband systems because of its great performance on circular polarization and maintaining consistent gain and input impedance. The Archimedean spiral antenna is a popular of frequency independent antenna. Previous wideband array designs with variable element sizes have used the Archimedean spiral antenna as the radiating element. The Archimedean spiral antenna radiates from a region where the circumference of the spiral equals one wavelength. This is called the active region of the spiral. The arm length is shorted by $46.4 \%$ compared to conventional Archimedean spiral antenna, and $63.5 \%$ compared to power spiral antenna.

A new spiral antenna structure is proposed in this paper. The antenna which is developed in this paper has a similar part to the outer circle and wraps in such a way that is not easy to move in the inner circle. The new spiral antenna has improved in axial ratio at low frequencies and better performance in high frequencies than power spiral, and the arm length is also very shorted.

Increasing use of wireless devices has led to the rise in the need of larger bandwidth so as to reduce interference. This need occurred due to the use of large differences in the frequencies used and a variety of frequency bands into existence. Since the bit rates are very high, there is a need for very high bandwidth to support wireless communication. In this paper, the simulation of frequency independent antenna - Spiral Antenna with the appropriate dimensions and frequency ranges and further the fabrication for the same, hence the proposed paper by paulino $\mathrm{N}$ et al [16] The simulation software involved is the HyperLynx IE3D software for the appropriate bandwidth ranges and dimensions. Widely used in defence industry, spiral antennas are antennas with a large bandwidth and very efficient in fast wireless communication. These wideband antennas are compact in size and do not occupy large space, thus easy to install. Basically there are two types of spiral antennas - the log periodic antenna and Archimedes antenna. In this paper they discussed the simulation of the Archimedes antenna. In this paper they suggested the implementation of the Archimedes antenna in the GPS applications. The antenna is circularly polarized antenna. There is a wide need for large 
frequency antennas because generally used antennas have a lower bandwidth and cannot transfer higher bit rates. In this paper an Archimedes spiral antenna operating at a frequency of $0.8 \mathrm{GHz}$ to $2.4 \mathrm{GHz}$ is simulated. Secondly, the matching circuits which are capable of adapting the impedance of the spiral antenna were also observed [7].

As spiral antennas transmit EM waves having circular polarization, it may be used in wideband communications. Another application of spiral antennas is monitoring of the frequency spectrum. Spiral antennas can be useful for microwave direction-finding.

Polarisation has always affected antenna's performance so considering this fact farzin lelzari et.al [17] in his work showcased the broadband dual polarized antenna consists of stacked spiral antenna arms which are electrically separated and each of them is orthogonal and has opposite senses. For the radiations by the lower spiral antenna to be degraded as low as possible, the relative overlap of spirals is kept minimum. Each pair of spiral arm shares a common axis with other pairs and also need not be co-planar. The spiral arm consists of spiralling wire elements. Such antennas can be used for beam scanning and shaping.

Thus on a concluding note the objective of the work is to provide a dual polarized antenna which is easy to manufacture and is simplified. The antenna manufactured can be used with airborne platforms and also will not interfere with the aerodynamics of an aircraft and have low observability characteristics.

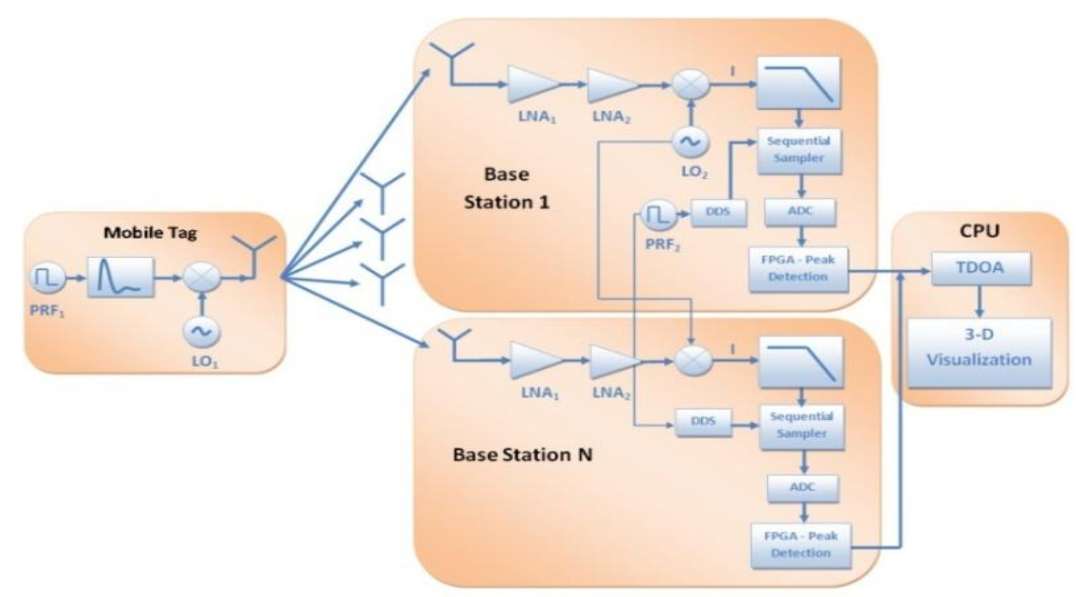

Figure3. Future of ultra band systems in medicine [21].

\section{CONCLUSION}

This paper deals with the advancements that have actually been made in the field of spiral antennas. The Chinese compass navigation system stressed on the importance of the fabrication process in the spiral antenna since the circular polarisation .The gain of spiral antenna can be increased considerably by forming an array. If we discuss about the feeding methods of a spiral antenna then the parallel feeding method proves to be the best. If the axial ratio is improved and arm length reduced then it may assist in improving the performance of antenna. Considering the fact that wireless communications require large bandwidth hence increasing importance of the frequency independent antennas ie, spiral antennas is pretty evident. Hence improving several parameters in a spiral antenna may lead to quite advancements in the related fields basically design optimisation has been the main aim of recent discoveries in the field of spiral antennas since they are quite compatible and serves as a merit for their increasing applications.

\section{ACKNOWLEDGEMENT}

We have taken efforts in this research work. However, it would not have been possible without the kind support and help of many individuals and organizations. We would like to extend our sincere thanks to all of them.

We would like to express our gratitude towards Hon. Chancellor SVKMs NMIMS; Management; Higher Authorities and Faculty Members for their kind co-operation and encouragement which helped us a lot. We would like to express our sincere thanks to industry persons and fellow colleagues for their kind attention and time. 


\section{REFERENCES}

[1] C. A. Balanis, Antenna Theory: Analysis and Design. New York: Wiley, 1982.

[2] Balanis, C.A. , Antenna theory: a review, Proceedings of the IEEE Volume:80 , Issue: 1 ,pp. 723,Jan 1992.

[3] Chinmaya vyas, Shashikant Patil, Amar khalore, comprehensive assessment of wearable smart textile sensors, International Journal of Advanced Information Science and Technology (IJAIST) ISSN: 2319:2682 Vol.45, No.45, January 2016.

[4] Orr, William I. (1976). Beam Antenna Handbook, 5th Edition. Radio Publications, Inc. pp. 185186.

[5] Paul E. Mayes, "Frequency-Independent Antennas and Broad-Band Derivatives Thereof", Proceedings of the IEEE, Volume: 80 , Issue: 1, Digital Object Identifier: 10.1109/5.119570, Publication Year: 1992, Page(s): 103 - 112)

[6] Huifen Huang and Zonglin Lv, A New Spiral Antenna with Improved Axial Ratio and Shorted Arm Length, Progress In Electromagnetics Research C, Vol. 46, 83-89, 2014.

[7] Stephen E. Lipsky "Microwave Passive Direction Finding" SciTech Publishing, 2004 ISBN 1891121-23-5 page 40.

[8] Paul E. Mayes, "Frequency-Independent Antennas and Broad-Band Derivatives Thereof', Proceedings of the IEEE, Volume: 80 , Issue: 1, Digital Object Identifier: 10.110915.119570, Publication Year: 1992,Page(s): 103 - 112.

[9] Muhammad Asad, Junaid GHani, Adnan Khalid and Mian Shahzad Iqbal, Optimizing Q Factor of Square Spiral Antenna, 2010 Second Pacific-Asia Conference on Circuits, Communications and System (PACCS).

[10] http://www.microwavejournal.com/articles/18897-spiral-antenna-designconsiderations? $\mathrm{v}=$ preview.

[11] Hangying Yuan, Shaobo Qu, Jieqiu Zhang, Hang Zhou, Jiafu Wang, Hua Ma, and Zhuo Xu, Dual-Band Dual-Polarized Spiral Antenna for Chinese Compass Navigation Satellite System, Progress In Electromagnetics Research Letters, Vol. 46, 25-30, 2014.

[12] N. H. Abdul Hadi, K. Ismail, S. Sulaiman and M. A. Haron, Design of a Rectangular Spiral Antenna for Wi-Fi Application, 13th International Conference on Advanced Communication Technology (ICACT), 2011 pg30-34,ISSN:1738-9445.

[13] Gschwendtner, E., J. Parlebas, and W. Wiesbeck, Spiral antenna with planar external feeding,"Proc. 29th Eur. Microwave Conf., Vol. 1, 135\{138, 1999.

[14] Huifen Huang and Zonglin Lv, A Spiral Antenna with Integrated Parallel-Plane Feeding Structure, Progress In Electromagnetics Research Letters, Vol. 45, 45-50, 2014.

[15] Magnus Karlsson, and Shaofang Gong, An integrated spiral antenna system for UWB, Microwave Conference, 2005 European (Volume:3 ) 4-6 Oct. 2005, DOI:10.1109/EUMC.2005.1610362.

[16] Paulino, N. Rebelo, H. ; Pires, F. ; Ventim Neves, I. ; Goes, J. ; Steiger-Garcao, A., Design of a Spiral-Mode Microstrip Antenna and Matching Circuitry for Ultra-Wide-Band Receivers, Circuits and Systems, 2002. ISCAS 2002. IEEE International Symposium on (Volume:3 ),pp 875-878, DOI:10.1109/ISCAS.2002.1010364.

[17] FarzinLalezari, Dual polarized spiral antenna,publication no US 5146234 A,

[18] Shashikant Patil, Arpan Patel and Ankur Bansal, Optimized Circularly Polarized Bandwidth for Microstrip Antenna, International Journal of Computing Academic Research (IJCAR) ISSN 2305-9184 Volume 1, Number 1 (October 2012), pp. 1-9.

[19] Prashant Sarode, Shashikant S. Patil, Sachin Sonawane, Girish Patil, A Novel design approach Microstrip Patch Antenna Design for Ultra wideband Applications, International Journal of Scientific \& Engineering Research, Volume 6, Issue 5, May-2015 1413 ISSN 22295518,pp1413-1416.

[20] http://www.antennamagus.com/newsletter-5-0.php

[21] Mohamed Mahfouz, Michael Kuhn and Gary to, the future of ultra wideband systems in medicine: orthopaedic surgical navigation, DOI: 10.5772/2050. 